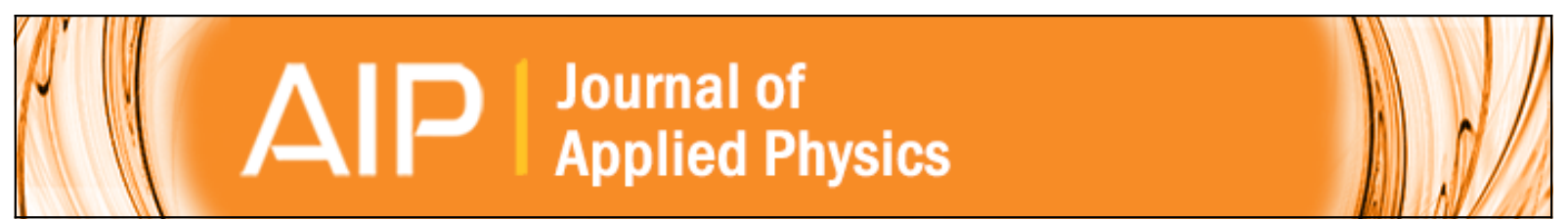

\title{
Structural dependence of nonlinear magnetoelectric effect for magnetic field detection by frequency modulation
}

Menghui Li, Yaojin Wang, Ying Shen, Junqi Gao, Jiefang Li, and D. Viehland

Citation: Journal of Applied Physics 114, 144501 (2013); doi: 10.1063/1.4824377

View online: http://dx.doi.org/10.1063/1.4824377

View Table of Contents: http://scitation.aip.org/content/aip/journal/jap/114/14?ver=pdfcov

Published by the AIP Publishing

\section{Articles you may be interested in}

Influence of metglas layer on nonlinear magnetoelectric effect for magnetic field detection by frequency modulation

J. Appl. Phys. 117, 024104 (2015); 10.1063/1.4905622

Frequency conversion in magnetoelectric composites for quasi-static magnetic field detection Appl. Phys. Lett. 103, 212902 (2013); 10.1063/1.4832861

Enhancement in magnetic field sensitivity and reduction in equivalent magnetic noise by magnetoelectric laminate stacks

J. Appl. Phys. 111, 104504 (2012); 10.1063/1.4718441

Dependence of magnetic field sensitivity of a magnetoelectric laminate sensor pair on separation distance: Effect of mutual inductance

J. Appl. Phys. 111, 033923 (2012); 10.1063/1.3684986

Enhanced dc magnetic field sensitivity by improved flux concentration in magnetoelectric laminates Appl. Phys. Lett. 99, 153502 (2011); 10.1063/1.3650713

MIT LINCOLN

LABORATORY CAREERS

Discover the satisfaction of innovation and service

to the nation
- Space Control

- Air \& Missile Defense

- Communications Systems \& Cyber Security

- Intelligence, Surveillance and

Reconnaissance Systems

- Advanced
Electronics
- Tactical Systems
- Homeland
Protection
- Air Traffic Control

LINCOLN LABORATORY

MassachusetTs Institute of TeChNOLOGY

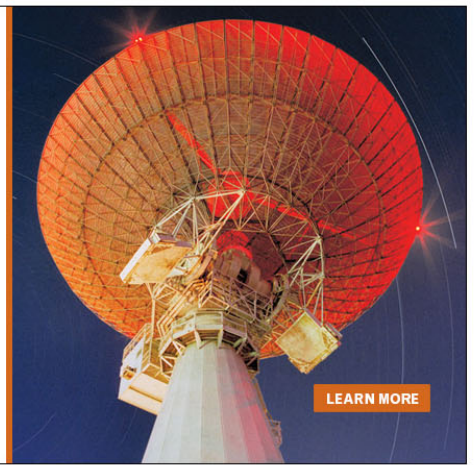




\title{
Structural dependence of nonlinear magnetoelectric effect for magnetic field detection by frequency modulation
}

\author{
Menghui Li, ${ }^{\text {a) }}$ Yaojin Wang, Ying Shen, Junqi Gao, Jiefang Li, and D. Viehland \\ Department of Materials Science and Engineering, Virginia Tech, Blacksburg, Virginia 24061, USA
}

(Received 21 August 2013; accepted 20 September 2013; published online 7 October 2013)

\begin{abstract}
The structure differences of magnetoelectric (ME) laminates for passive and active mode sensors are discussed. The Fourier coefficient $A_{1}$ calculated from the data of $\alpha_{\mathrm{ME}}-H_{\mathrm{dc}}$ indicates that $N=1$ (where $N$ is the number of Metglas layers) should be the optimum structure for the active mode. Experimental investigations of the magnetic field sensitivity agree well with this conjecture. For $N=1$, the magnetic field sensitivity was $0.66 \mathrm{nT} / \mathrm{Hz}^{0.5}$, which was 3.1 times larger than for $N=5$. (C) 2013 AIP Publishing LLC. [http://dx.doi.org/10.1063/1.4824377]
\end{abstract}

The magnetoelectric (ME) effect-the induction of magnetization by an applied electric field $(E)$ or polarization by magnetic field $(H)$ - has been of recent research interests due to many potential applications. ${ }^{1-3}$ The ME effect in laminate composites is known to be much larger than in single phase and particulate composites, due to a combination of the magnetostrictive and piezoelectric effects of the individual layers. ${ }^{4-6}$ Application of magnetic field to the ME laminates produces an elastic strain in the magnetostrictive phase that is then stress coupled to that of the piezoelectric layer, resulting in an induced voltage. Significantly, higher values of the ME voltage coefficient $\left(\alpha_{M E}\right)$ have been reported in tri-layer Metglas/piezo-fibers/Metglas structures with a multi-push-pull configuration that is longitudinally poled and longitudinally magnetized (L-L), promising applications such as magnetic field sensors and gradiometers. ${ }^{7}$

In previous reports, motivated by passive magnetic sensor applications, a linear response of the ME composites to a weak AC magnetic field $H_{\mathrm{AC}}$ was shown. ${ }^{8}$ A DC magnetic bias field was applied to obtain the maximum piezomagnetic coefficient $d_{33, \mathrm{~m}}$, yielding the highest induced ME voltage $\left(V_{\mathrm{ME}}\right)$ to $H_{\mathrm{AC}}$. Recently, ME laminate composites have also been used in an active mode to sense small DC magnetic fields or weak AC magnetic fields, via a frequency modulation technique. ${ }^{9,10}$ Such an approach has been utilized to reduce the environmental vibrational noise and $1 / f$ noise in the low frequency range. In a magnetically unshielded environment, the sensitivity to small changes in $H_{\mathrm{AC}}$ can be enhanced by at least two orders magnitude compared to passive magnetic sensors. ${ }^{10}$ However, to date, all investigations of the nonlinear ME coefficient, which is the physical basis of the frequency modulation method, have been reported using the same laminate structure as that used in the passive mode. ${ }^{11}$ Due to a dependence of the different orders of nonlinearity on the magnetostrictive material, the structure resulting in a maximum nonlinear ME coefficient may not be the same as for the linear ME effect used in the passive mode. However, any investigations of the ME laminate structure that optimize the mode have not yet been performed.

a)limh09@vt.edu
In this letter, the structure differences of ME laminates for sensor applications in passive and active modes are discussed. Theoretical predictions and experimental results both illustrate that $N=1$ is the optimum structure for the active ME sensors. For $N=1$, the magnetic field sensitivity was 3.1 times larger than for $N=5$.

Several Metglas/PZT/Metglas multi-push-pull L-L mode laminates were made. ${ }^{12}$ A $40 \mathrm{~mm} \times 10 \mathrm{~mm}$ PZT bundle served as the core, which consisted of five $40 \mathrm{~mm} \times 2 \mathrm{~mm}$ PZT-5A fibers (Smart Materials, Sarasota, FL) oriented along the length direction of the laminates. Two interdigitated (ID) Kapton electrodes were bonded to the top and bottom surfaces of the piezoelectric bundle with an epoxy resin (Stycast 1264, USA). The width and the separation of the electrodes were chosen to be $0.15 \mathrm{~mm}$ and $1 \mathrm{~mm}$, respectively. Different numbers $N$ of Metglas layers (Vitrovac 7600F, Hanau, German) of dimensions $80 \mathrm{~mm} \times 10 \mathrm{~mm}$ were bonded to both sides of the PZT core composite for $N=1$ to 7 .

The value of $\alpha_{\mathrm{ME}}$ was then measured using a lock-in amplifier (SR-850) in response to a pair of Helmholtz coils driven at an $\mathrm{AC}$ magnetic field of $H_{\mathrm{AC}}=0.1 \mathrm{Oe}$ at a frequency of $f=1 \mathrm{kHz}$. A DC magnetic field was applied by a large electromagnet. Figure 1(a) shows the values $\alpha_{\mathrm{ME}}$ as a function of $H_{\mathrm{DC}}$ for Metglas/PZT/Metglas laminates with different $N$. It can be seen that $\alpha_{\mathrm{ME}}$ increased as $H_{\mathrm{DC}}$ was increased; and subsequently decreased as $H_{\mathrm{DC}}$ was further increased. The maximum value of $\alpha_{\mathrm{ME}}$ increased with increasing number of Metglas layers until $N=5$, and then decreased with further increase in $N$. For the optimum value of $N$, the magnetic field sensitivity of the passive ME sensor was about $20 \mathrm{pT} / \mathrm{Hz}^{0.5} \cdot 13,14$

For a constant applied AC magnetic field, the output voltage $V_{\mathrm{ME}}$ is proportional to $\alpha_{\mathrm{ME}}$. The value of $V_{\mathrm{ME}}$ can be then given as a five-order Fourier expansion series, ${ }^{15}$ as

$$
V_{M E}=a_{0}+a_{1} H+a_{2} H^{2}+a_{3} H^{3}+a_{4} H^{4}+a_{5} H^{5},
$$

where $H=H_{\text {mod }} \cos \left(2 \pi f_{\text {mod }} t\right)$ is an applied AC magnetic field, $H_{\text {mod }}$ is the modulation amplitude, and $a_{i}(i=0,1,2 \ldots)$ are the $i^{\text {th }}$ order Fourier coefficients of the field. The values of $a_{i}$ can be estimated by fitting (1) to the $\alpha_{\mathrm{ME}}-H_{\mathrm{dc}}$ data of Fig. 1(a), as shown in the Appendix for $N=1$ to 7 . At the 

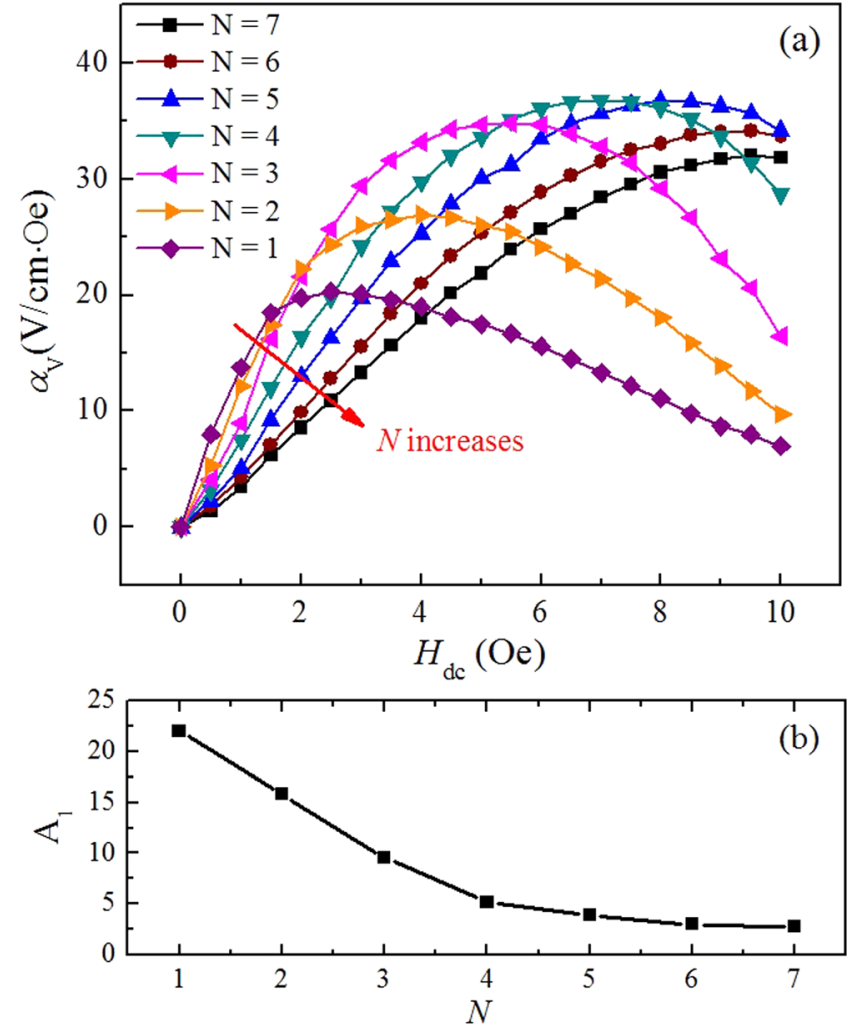

FIG. 1. (a) $\alpha_{\mathrm{ME}}$ as a function of $H_{\mathrm{dc}}$ for Metglas/PZT/Metglas laminates with different values of $N$, and (b) values of $A_{1}$ calculated by Eq. (2) for various $N$.

modulation frequency $f_{\text {mod }}, V_{\mathrm{ME}}$ is proportional to a rearranged Fourier coefficient $A_{1}$, given as

$$
A_{1}=a_{1} H_{\mathrm{mod}}+\frac{3}{4} a_{3} H_{\mathrm{mod}}^{3}+\frac{5}{8} a_{5} H_{\mathrm{mod}}^{5} .
$$

Figure 1(b) shows the calculated values of $A_{1}$ for various $N$. With increasing $N$, the value of $A_{1}$ decreases, implying for $N=1$ that $V_{\mathrm{ME}}$ is the highest. These results illustrate that the optimum structure of Metglas/PZT/Metglas laminate for the active mode is different than that for the passive one.

Next, the $M-H$ hysteresis loops were measured by a vibrating sample magnetometer (VSM) for various values of $N$, as shown in Figure 2(a). The value of the magnetic susceptibility $\chi$ was then calculated as $d M_{\mathrm{s}} / d H$, where $\mathrm{M}_{\mathrm{s}}$ is the saturation magnetization. In Figure 2(b), the value of $\chi$ can be seen to be maximum for $N=1$, and to decrease as $N$ increases. This decrease was due to the constraint imposed by epoxy bonding layers between the Metglas layers. As $N$ is increased, the additional epoxy layers increasingly constrain the magnetostriction of the Metglas layers. Such constraint makes the longitudinal magnetization more difficult. ${ }^{16}$ To achieve an optimum magnetostriction, the value of the required $H_{\mathrm{DC}}$ has to be increased as $N$ is increased. Thus, $\chi$ effectively decreases with increasing $N .{ }^{17}$ For an AC modulation field $H_{\text {mod }}$ and a small signal field $H_{\mathrm{AC}}$, the magnetostriction $\lambda$ of the Metglas layers at the frequency of $f_{\text {mod }}-f_{A C}$ can then be given as ${ }^{18}$
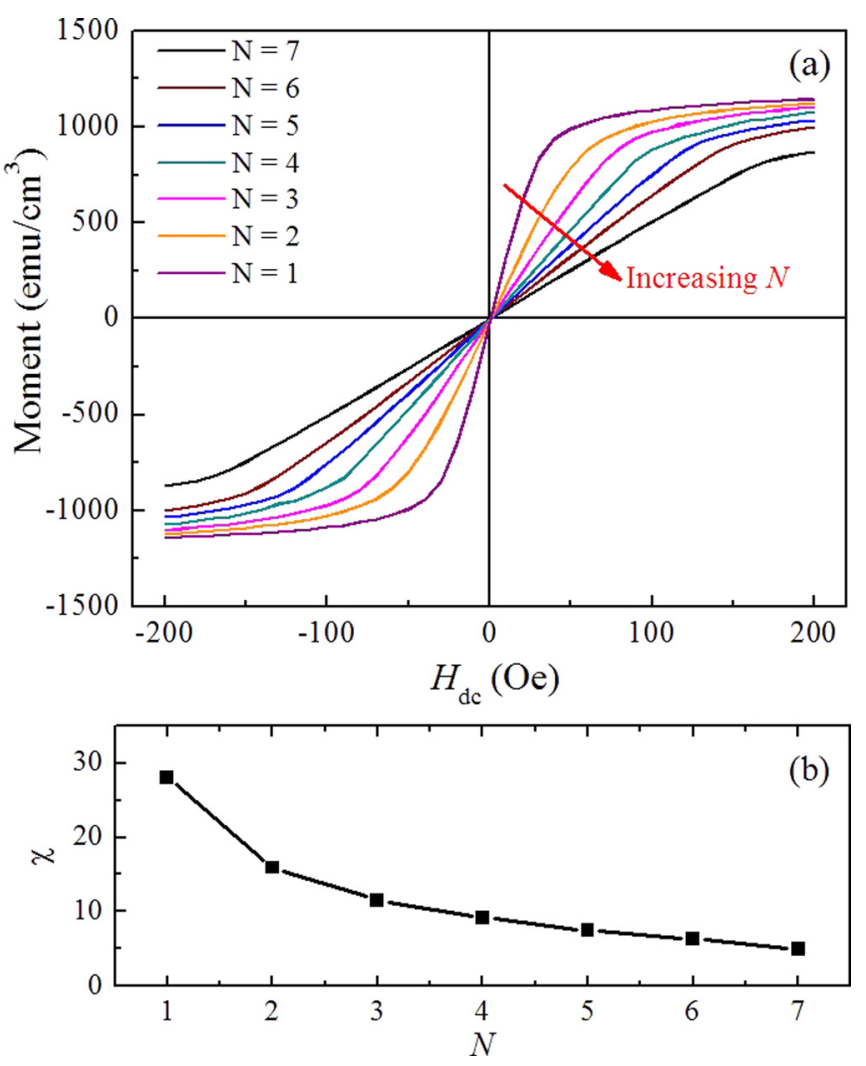

FIG. 2. (a) $M-H$ hysteresis loops and (b) $\chi$ for various values of $N$.

$$
\lambda=\frac{3 \lambda_{s} \chi^{2}}{2 M_{s}^{2}} H_{\mathrm{mod}} H_{A C}
$$

where $\lambda_{s}$ is the saturation magnetostriction. From (3), it can be seen that $V_{\mathrm{ME}}$ is directly proportional to $\chi^{2}$. Thus, to get the highest value of the output signal, the structure that yields the maximum value of $\chi$ should be chosen, which is $N=1$.

Finally, an incident magnetic field of $H_{\text {mod }}=1$ Oe at $f_{\text {mod }}=1 \mathrm{kHz}$ was applied using a Helmholtz coil and an AC field of $B_{\mathrm{AC}}=70 \mathrm{nT}$ at a $f_{A C}=1 \mathrm{~Hz}$ using a drive coil. The modulation field $H_{\text {mod }}$ was then measured using a SR-785 dynamic signal analyzer (Stanford Research Systems). Figure 3(a) shows the measured modulation spectrum in the frequency range between $998.5 \mathrm{~Hz}$ and $1001.5 \mathrm{~Hz}$ for various $N$. As shown in Figure 3(b), for $N=1$, the ME output voltage $V_{\mathrm{ME}}$ at $f_{\text {mod }}-f_{A C}$ was maximum; and subsequently decreased with increasing $N$. At $f=f_{\text {mod }}-f_{A C}$, the voltage noise $V_{\text {noise }}$ for $B_{\mathrm{AC}}=0$ at the value of each $N$ was also measured as shown on the right axis of Fig. 3(b). With increasing $N, V_{\text {noise }}$ also increased slightly, due to an increased value of $\alpha_{\mathrm{ME}}$ at low fields (i.e., $H_{\mathrm{DC}}<1 \mathrm{Oe}$ ). The magnetic field sensitivity can be estimated as $V_{\mathrm{ME}} / B_{\mathrm{AC}} * V_{\text {noise}}$, as shown in Figure 3(c). With increasing $N$, the active sensor had an enhanced magnetic field sensitivity. The magnetic field sensitivity was $0.66 \mathrm{nT} / \mathrm{Hz}^{0.5}$ for $N=1$, which was 4.2 times larger than for $N=7$ and 3.1 times larger than for $N=5$. Clearly, the optimum structure for the $\mathrm{ME}$ active mode structure $(N=1)$ is different than the passive one $(N=5)$. The increased background noise by modulation mainly limits the sensitivity. ${ }^{10,15}$ 

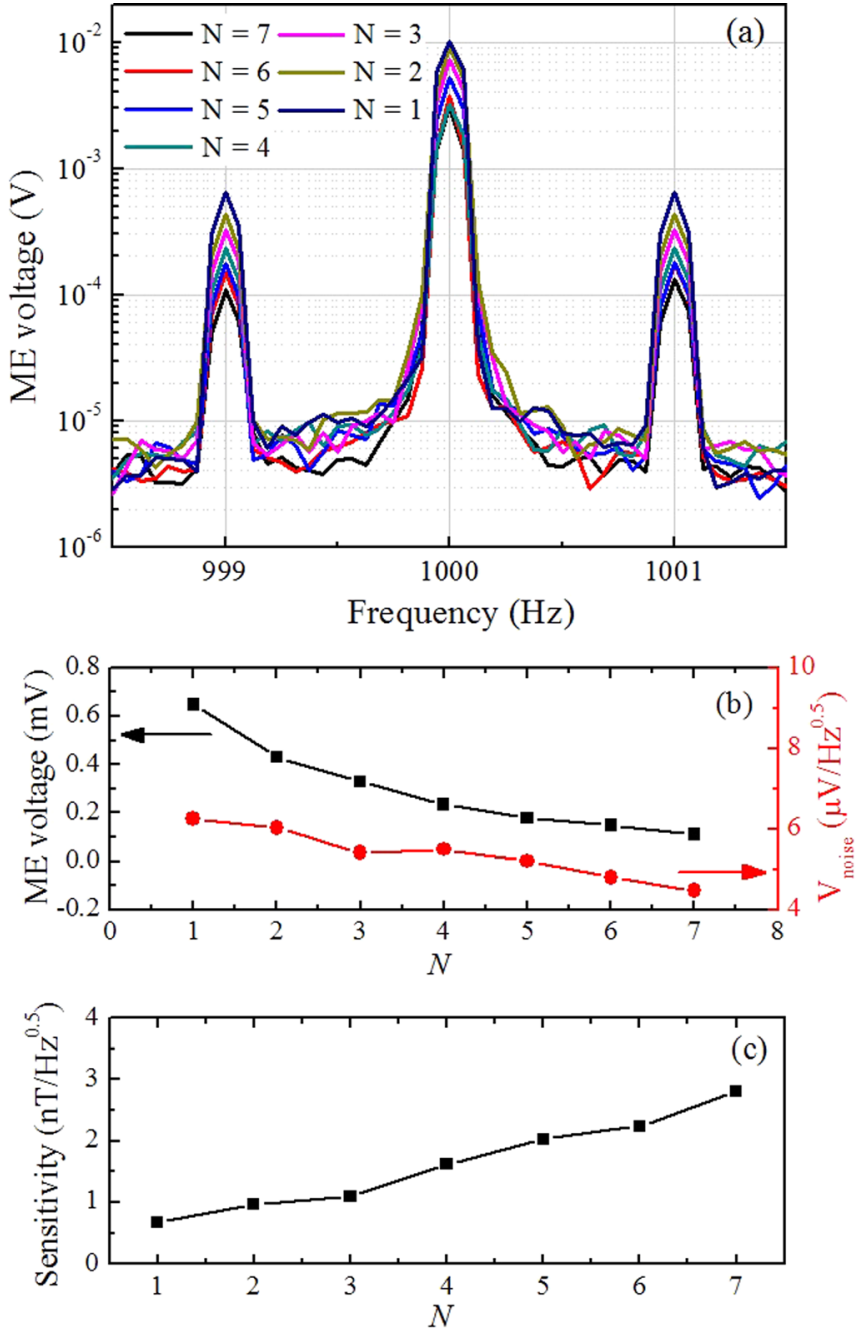

FIG. 3. (a) Measured modulation spectrum taken over the frequency of $998.5 \mathrm{~Hz}$ to $1001.5 \mathrm{~Hz}$ for various values of $N$; (b) $V_{\mathrm{ME}}$ and $V_{\text {noise }}$ at $f=f_{\text {mod }}-f_{A C}$; and (c) magnetic field sensitivity as a function of $N$.

If the undesired background noise could be reduced, the sensitivity could be further enhanced by two orders of magnitudes.

In conclusion, we revealed the structure differences of ME laminate for sensor applications in passive and active modes. In active mode, the calculated Fourier coefficient $A_{1}$ indicates that the $\mathrm{ME}$ output voltage is highest for $N=1$. Experimental results agree well with the prediction, and are explained by the change of $\chi$. The magnetic field sensitivity is enhanced by optimizing structure.
This work was supported by the Office of Naval Research.

\section{APPENDIX: ESTIMATED FOURIER COEFFICIENTS AI FROM FIGURE 1(A)}

\begin{tabular}{lccccccc}
\hline \hline $\mathrm{N}$ & 1 & 2 & 3 & 4 & 5 & 6 & 7 \\
\hline$a_{0}$ & -0.27 & -0.76 & -0.7 & -0.06 & -0.17 & -0.03 & -0.13 \\
$a_{1}\left(\mathrm{Oe}^{-1}\right)$ & 21.1 & 15.8 & 10.2 & 5.7 & 4.25 & 3.21 & 2.94 \\
$a_{2}\left(\mathrm{Oe}^{-2}\right)$ & -7.76 & -2.51 & 1.57 & 2.31 & 1.98 & 1.43 & 1.08 \\
$a_{3}\left(\mathrm{Oe}^{-3}\right)$ & 1.3 & 0.02 & -0.8 & -0.71 & -0.54 & -0.33 & -0.24 \\
$a_{4}\left(\mathrm{Oe}^{-4}\right)$ & -0.11 & 0.019 & 0.091 & 0.071 & 0.053 & 0.027 & 0.021 \\
$a_{5}\left(\times 10^{-3} \mathrm{Oe}^{-5}\right)$ & 3.50 & -1.10 & -3.50 & -2.60 & -1.90 & -0.896 & -0.752 \\
\hline \hline
\end{tabular}

${ }^{1}$ N. A. Spaldin and M. Fiebig, Science 309, 391 (2005).

${ }^{2}$ R. Ramesh and N. A. Spaldin, Nature Mater. 6, 21 (2007).

${ }^{3}$ C.-W. Nan, M. I. Bichurin, S. Dong, D. Viehland, and G. Srinivasan, J. Appl. Phys. 103, 031101 (2008).

${ }^{4}$ S. Dong, J. Zhai, J. Li, and D. Viehland, Appl. Phys. Lett. 89, 252904 (2006).

${ }^{5}$ J. Zhai, Z. Xing, S. Dong, J. Li, and D. Viehland, J. Am. Ceram. Soc. 91, 351 (2008).

${ }^{6}$ M. Li, Y. Wang, D. Hasanyan, J. Li, and D. Viehland, Appl. Phys. Lett. 100, 132904 (2012).

${ }^{7}$ Y. Wang, D. Gray, D. Berry, J. Gao, M. Li, J. Li, and D. Viehland, Adv. Mater. 23, 4111 (2011).

${ }^{8}$ S. Dong, J. Zhai, Z. Xing, J.-F. Li, and D. Viehland, Appl. Phys. Lett. 86, 102901 (2005).

${ }^{9}$ M. L. C. S. X. Zhuang, C. Cordier, S. Saez, C. Dolabdjian, L. Shen, J. F. Li, M. Li, and D. Viehland, "Evaluation of applied axial field modulation technique on ME sensor input equivalent magnetic noise rejection," IEEE Sens. J. 11, 2266 (2011).

${ }^{10}$ J. Petrie, D. Gray, D. Viehland, G. Sreenivasulu, G. Srinivasan, S. Mandal, and A. S. Edelstein, J. Appl. Phys. 111, 07C714 (2012).

${ }^{11}$ L. Shen, M. Li, J. Gao, Y. Shen, J. F. Li, D. Viehland, X. Zhuang, M. Lam Chok Sing, C. Cordier, S. Saez, and C. Dolabdjian, J. Appl. Phys. 110, 114510 (2011).

${ }^{12}$ M. Li, D. Berry, J. Das, D. Gray, J. Li, and D. Viehland, J. Am. Ceram. Soc. 94, 3738 (2011).

${ }^{13}$ M. Li, Y. Wang, J. Gao, J. Li, and D. Viehland, Appl. Phys. Lett. 101, 022908 (2012).

${ }^{14}$ M. Li, D. Hasanyan, Y. Wang, J. Gao, J. Li, and D. Viehland, J. Phys. D: Appl. Phys. 45, 355002 (2012).

${ }^{15}$ R. Jahns, H. Greve, E. Woltermann, E. Quandt, and R. Knöchel, Sens. Actuators, A 183, 16 (2012).

${ }^{16}$ X. J. Zheng and X. E. Liu, J. Appl. Phys. 97, 053901 (2005).

${ }^{17}$ F. Fang, C. Zhao, and W. Yang, Sci. China, Ser. G 54, 581 (2011).

${ }^{18}$ A. L. G. M. Gillette, D. Gray, D. Viehland, C. Vittoria, and V. G.Harris, "Improved sensitivity and noise in magneto-electric magnetic field sensors by use of modulated AC magnetostriction," IEEE Magn. Lett. 2, 2500104 (2011). 